\title{
Digestibilidad asociada de ramio (Boehmeria nivea graud) en dietas acompañadas con concentrados en conejos
}

\section{Digestibility associated ramie (Boehmeria nivea graud) in diets with concentrates in rabbits}

\author{
Burgos D, Virviéscas $G$, Peña $F$, Pérez $M$, Benítez $M^{1}$ y Roa $M L^{2}$ \\ ${ }^{1}$ Estudiantes de Medicina Veterinaria y Zootecnia y \\ 2Docente: Escuela de Ciencias Animales Universidad de los Llanos
}

\section{dabudu19@hotmail.com}

Recibido 16 de junio 2011 aceptado 19 de septiembre 2011

\section{RESUMEN}

El experimento se desarrolló en la granja Barcelona de la Universidad de los Llanos, el objetivo fue determinar la digestibilidad in vivo del ramio (Boehmeria nivea graud), para demostrar el aporte nutricional de este forraje. Se utilizaron 16 conejos, peso promedio $3.200 \pm 100$ gramos, divididos en 4 grupos que fueron alojados en jaulas, las dietas suministradas fueron: Tratamiento 1: 100\% de la dieta fue ramio ad libitum (T1), Tratamiento 2 : Ramio ad libitum y $0.5 \%$ del peso vivo (PV) en concentrado comercial (CC) (T2), Tratamiento 3: Ramio ad libitum y $1 \%$ del PV en CC (T3) y Tratamiento 4: Ramio ad libitum y $1,5 \%$ del PV en CC (T4). Se tomaron datos durante 14 días de los cuales cuatro días fueron de adaptación del alimento y diez de recolección de muestras. El diseño fue completamente al azar con diez repeticiones y 4 tratamientos. Las variables evaluadas fueron: consumo de forrajes, excreción por heces, digestibilidades: Materia seca (MS), proteína, grasa, extracto no nitrogenado (ENN) y fibra cruda (FC), y \% de nutrientes digestibles totales (NDT) y cálculos de: Energía digestible (ED) y energía metabolizable (EM). Las digestibilidades asociadas de la materia seca, la proteína y ENN fueron menores $(p<0.05)$ en $T 1$, mientras que en la grasa no se observó diferencias entre tratamientos. Se demostró que el aumento de concentrado tiene una relación directamente proporcional con al incremento del porcentaje de NDT y la digestibilidad asociada de la MS y la proteína.

Palabras clave: Digestión, lepóridos, forraje herbáceo. 


\begin{abstract}
The experiment was developed in the farm Barcelona of the Llanos University, the objective was to determine the in vivo digestibility Boehmeria nivea graud to demonstrate the nutritional quality of this forage. Sixteen rabbits were used, with average weight $3.200 \pm 100$ grams, they were divided into 4 groups, they were housed in cages, the diets fed were: Treatment 1: 100\% B. nivea diet was ad libitum (T1), Treatment 2: B. nivea ad libitum and $0.5 \%$ of body weight (BW) in commercial concentrate (CC) (T2), Treatment 3: B. nivea ad libitum and $1 \%$ of body weight (BW) (T3) and Treatment 4: B. nivea ad libitum and $1.5 \%$ of body weight (BW) (T4). The Dates were collected during 14 days of which were four days of food adaptation and ten sample collections. The design was completely randomized with ten replications and 4 treatments. The variables evaluated were: forage intake, feces excretion, digestibility: Dry matter (DM), protein, fat, nitrogenfree extract (NFE), crude fiber (CF), \% total digestible nutrients (TDN) and calculations: digestible energy (DE) and metabolizable energy (ME). The associated digestibilities of dry matter, protein and NFE were lower $(p<0.05)$ in T1, while the fat was similar in all treatments. It was shown that the increase in concentrate has a directly proportional relationship with the increase in the percentage of NDT and the associated digestibility of DM and protein.
\end{abstract}

Keywords: Digestion, leporid, hebaceous forage.

\title{
INTRODUCCIÓN
}

La producción animal eficiente y con bajos costos conlleva a una selección de materias primas con alta biodisponibilidad que compita lo menos posible con la alimentación humana. Cada materia prima debe tener una evaluación completa de sus características fisicoquímicas que contribuya a determinar los efectos fisiológicos en el animal. La investigación en nutrición animal se ha enfocado a la búsqueda de metodologías que aumenten los indicadores productivos, uno de los objetivos es el conocimiento de los requerimientos para obtener sistemas producción competitivos. La cunicultura en países tropicales, constituye una 
opción interesante para producción de carne con elevado valor nutricional para la dieta humana, se puede considerar como una estrategia válida para mejorar las condiciones de vida en áreas rurales socioeconómicamente deprimidas, donde puede enfocarse para autoconsumo y generación de ingresos (Preston, 1998; Nieves et al., 2004).

El desarrollo del experimento permite determinar diferentes aspectos de la utilización del ramio (Boehmeria nívea graud), en alimentación de conejos, además de mostrar el aporte nutricional de este forraje. En el experimentó se determinó la composición nutricional de este forraje para comparar el efecto de varios niveles de inclusión sobre la digestibilidad de la materia seca, proteína cruda y energía con respecto a los diferentes resultados obtenidos con el uso de concentrado comercial en la alimentación de conejos. Posterior a la obtención de estos resultados se realizó una comparación de estas evaluaciones cuando se usa concentrado, identificando sus beneficios en la respuesta productiva de los animales. Los estudios de valoración nutricional son útiles para determinar el contenido de energía y proteína digestible que deben ser usados para un ingrediente en formulación de raciones. La energía digerible es el valor más extensamente utilizado en evaluación de energía de alimentos para conejos debido a su estrecha relación con la energía metabolizable y a su simplicidad (De Blas et al., 1985).

Los requerimientos nutricionales en el conejo son altos en comparación con otras especies animales no rumiantes (De Blas et al., 1985), esta condición convierte al ramio (Boehmeria nívea graud) en una fuente importante de proteína y de fácil adaptabilidad a diferentes condiciones medio ambientales que puede ser utilizada como suplemento en la dieta de todos los animales tanto no rumiantes como en rumiantes. Es una de las especies arbóreas de mayor palatabilidad, que se adapta desde los 400 a $1.500 \mathrm{msnm}$, son pocos los estudios relacionados a la fecha que incluyan la evaluación química y nutricional de este forraje para una posterior comparación con el uso de concentrados comerciales como fuente de alimentación en conejos. El elevado contenido proteínico de las hojas, determinó 
que investigadores de países tropicales y subtropicales (Guatemala, Brasil, Sur de EE.UU.) estudiaran su aptitud forrajera, considerándola una planta de alto potencial alimenticio por la producción y calidad nutricional. En distintas experiencias con bovinos, ovinos, porcinos, equinos y aves, esta especie probó la factibilidad de ser utilizada como recurso nutricional bajo la forma de forraje verde y/o harina, (Cheeke, 1987; Jenkings, 1999).

Existen diferentes características que el alimento para conejos debe cumplir con respecto a sus características físico-químicas, el conejo necesita muy poca grasa (3.5\%), los alimentos que consume generalmente le aportan la cantidad necesaria, la fibra y la proteína dependerá del estado fisiológico: Gazapos en crecimiento 13$17 \%$, madres lactantes $17-18 \%$, crecimiento y engorde $13-14 \%$. Respecto a las sales minerales son importantes algunos elementos como calcio, fósforo, cloro y sodio, también magnesio, hierro, cobre, zinc y yodo, éstas se adicionan generalmente en un suplemento mineral en un total de $1 \%$ de la dieta (Nieves et al., 2006).

La búsqueda de nuevas alternativas nutricionales para conejos de producción permite desarrollar investigaciones que estén encaminadas a la búsqueda de nuevas fuentes de alimentos que cumplan con las necesidades nutricionales del animal en cada una de sus etapas fisiológicas. La determinación del valor nutricional de fuentes no convencionales debe realizarse mediante la utilización de métodos que permitan obtener el máximo de información acerca de las características nutritivas en el menor tiempo y de la forma más económica posible (Nieves et al., 2008).

El análisis del aprovechamiento de cada uno de los nutrientes en el organismo del animal se hace teniendo en cuenta su digestibilidad, ésta valoración se consigue con diferentes métodos. La digestibilidad in vivo de un alimento se puede medir directa e indirectamente. En la forma directa se registra exactamente el consumo de alimento y la excreción fecal de un animal sometido a un tratamiento dietético, en un período de tiempo dado. Como desventaja de este método, puede existir contaminación entre excretas y orina; además el confinamiento de los animales 
reduce el tono muscular y probablemente al disminuir el tránsito de ingesta, se sobreestima la digestibilidad con respecto a los animales no alojados en jaulas. La forma indirecta para medir la digestibilidad no requiere cuantificar el consumo ni la excreción fecal, se puede utilizar marcadores que se agregan o que están incluidos en el alimento (Nieves et al., 2008). El desarrollo de la digestibilidad comparada permite encontrar la relación del aprovechamiento de la proteína en el animal, partiendo de una comparación entre las nuevas alternativas de alimentación y el concentrado comercial comúnmente utilizado en la cunicultura.

Curch y Pond, (1990) y Castellanos et al., (1990) afirman que el método de los nutrientes digestibles totales (NDT) valora el alimento en su contenido de energía, partiendo de los cálculos de digestibilidad directa in vivo, donde se mide el nutriente consumido (NC) y el excretado (NE), realizando los análisis proximales para aplicar fórmulas para determinar los coeficientes de digestibilidad de cada nutriente (cod). Al contenido de carbohidratos digestibles, llamado extracto no nitrogenado digestible (ENN), se le suma las fracciones digestibles de la fibra, proteína y grasa (ésta última multiplicada por la constante 2.25), se obtiene el valor energético relativo de un ingrediente. Además, la equivalencia de $1 \mathrm{Kg}$ de $\mathrm{NDT}=4.400 \mathrm{Kcal}$ Energía Digestible ó a 3.560 Kcal Energía Metabolizable.

Coeficiente de digestibilidad de un nutriente $(\operatorname{Cod})=\frac{N C-N E}{N C * 100}$

$\% \boldsymbol{N D T}=(\%$ de prot $* \operatorname{cod})+(\%$ de grasa $* \operatorname{cod} * 2.25)+(\%$ de fibra $* \operatorname{cod})$ $+(\%$ de ENNx cod $)$

En un ensayo reportado por Church y Pond, (1990) se estudiaron diferentes métodos de evaluación de las digestibilidades comparadas de alimentos concentrados y forrajes. Se observó que ciertas fracciones como la fibra cruda, el extracto libre de nitrógeno y la grasa, se pueden sobrevalorar con el cálculo de la digestibilidad asociativa por diferencia. A pesar de esto, es un dato cercano a la digestibilidad total de una ración constituida por forraje y concentrado, aunque no refleje en su totalidad la verdadera digestibilidad de éste, pero sí se ha 
comprobado que en las dietas compuestas por varios ingredientes se presenta el fenómeno de la digestibilidad asociativa.

\section{MATERIALES Y MÉTODOS}

El experimento se desarrolló en la granja Barcelona y el laboratorio de Nutrición Animal de la Facultad de Ciencias Agropecuarias y Recursos Naturales de la Universidad de los Llanos en la ciudad de Villavicencio, Meta región de la Orinoquia zona de piedemonte, con una altitud de 465 metros sobre el nivel del mar, una temperatura promedio de $27^{\circ} \mathrm{C}$ y una precipitación anual entre 1.900 y $2.300 \mathrm{~mm}$ (Roa et al., 1999). Se utilizaron 16 conejos, peso promedio $3.200 \pm 100$ gramos, divididos en 4 grupos que fueron alojados en jaulas de alambre galvanizado de $0,30 \times 0,10 \times 0,20 \mathrm{~m}$, las cuales estaban dotadas de comederos tubulares y bebederos tipo chupón; éste diseño de las jaulas permitía recoger las excretas para el posterior análisis. El ramio y el concentrado comercial se analizaron (Tabla 1), para determinar su calidad nutricional antes de empezar el experimento (AOAC, 2006). Las dietas se suministraron por 14 días de los cuales cuatro días fueron de adaptación del alimento y 10 de recolección de muestras. Las dietas suministradas fueron: Tratamiento 1: 100\% de la dieta fue ramio ad libitum (T1), Tratamiento 2: Ramio ad libitum y $0.5 \%$ del peso vivo (PV) en concentrado comercial (CC) (T2), Tratamiento 3: Ramio ad libitum y $1 \%$ del PV en CC (T3) y Tratamiento 4: Ramio ad libitum y $1,5 \%$ del PV en CC (T4).

Se aplicó un diseño completamente al azar con diez repeticiones y 4 tratamientos. Las variables evaluadas fueron: consumo de forrajes, excreción por heces, digestibilidades: Materia seca (MS), proteína, grasa, extracto no nitrogenado (ENN), fibra cruda (FC), y \% NDT; y cálculos de: Energía digestible (ED) y energía metabolizable (EM). (Church y Pond, 1990, y Nieves et al., 2008). Se realizó un análisis de varianza, y haciendo una prueba de comparación múltiple Duncan.

\section{RESULTADOS Y DISCUSIÒN}

Los análisis nutricionales de las excretas (Tabla 2), muestran que T1 fue el mayor porcentaje de materia seca (MS) y extracto no nitrogenado (ENN), además el de 
mayor excreción de MS $(p<0.05)$ y de proteína en comparación con los demás tratamientos (Tablas 3 y 4). T4 obtuvo el menor porcentaje de MS, proteína y grasa, lo mismo sucedió con la excreción de la materia seca y la proteína, las cuales fueron las más bajas $(\mathrm{p}<0.05)$ con relación a los otros tres tratamientos (Tablas 3 y 4). Lo cual indica que el consumo y el contenido nutricional de las raciones afecta la cantidad de excretas y su composición, debido a la dinámica digestiva de cada nutriente y a la asociación de los ingredientes de la dieta (Nieves et al., 2001; 2002).

Tabla 1. Composición nutricional (\%) de los constituyentes de los tratamientos

\begin{tabular}{|c|c|c|} 
Nutrientes & Concentrado & Ramio \\
\hline Materia Seca & 2.1 & 47.1 \\
\hline Proteína & 13.48 & 19.15 \\
\hline Grasa & 10.3 & 9.2 \\
\hline Fibra cruda & 13.1 & 11.3 \\
\hline ENN & 55.99 & 49.44 \\
\hline Cenizas & 8.73 & 9.53 \\
\hline
\end{tabular}

Fuente: Laboratorio de Nutrición Animal de UNILLANOS

Tabla 2. Análisis proximal (\%) de Excretas en los tratamientos

\begin{tabular}{|l|c|c|c|c|} 
Nutrientes & $\mathrm{T} 1$ & $\mathrm{~T} 2$ & $\mathrm{~T} 3$ & $\mathrm{~T} 4$ \\
\hline Materia seca & 39.57 & 36.11 & 33.90 & 30.6 \\
\hline Proteína & 8.01 & 11.58 & 11.30 & 6.48 \\
\hline Grasa & 4.32 & 3.80 & 4.10 & 3.51 \\
\hline Fibra cruda & 4.01 & 7.80 & 6.90 & 7.1 \\
\hline ENN & 73.09 & 64.37 & 65.46 & 70.57 \\
\hline
\end{tabular}

Tabla 3. Consumo de alimento y excreción/ día en gramos de materia seca

\begin{tabular}{|c|c|c|c|c|}
\hline Parámetros & $\mathrm{T} 1$ & $\mathrm{~T} 2$ & $\mathrm{~T} 3$ & $\mathrm{~T} 4$ \\
\hline Consumo concentrado & 0 & 16.00 & 32.00 & 48.00 \\
\hline Consumo ramio & $86.11^{\mathrm{d}}$ & $68.56^{\mathrm{c}}$ & $43.24^{\mathrm{b}}$ & $24.43^{\mathrm{a}}$ \\
\hline Consumo total & $86.11^{\mathrm{b}}$ & $84.56^{\mathrm{b}}$ & $75.84^{\mathrm{a}}$ & $74.43^{\mathrm{a}}$ \\
\hline Excreción total & $20.5^{\mathrm{c}}$ & $15.35^{\mathrm{b}}$ & $13.45^{\mathrm{ab}}$ & $10.34^{\mathrm{a}}$ \\
\hline
\end{tabular}

Letras distintas en la misma fila son diferentes $(p<0.05)$.

La digestibilidad asociada de la MS en T4 fue superior $(p<0.05)$ en comparación con T1 y T2, éste mismo tratamiento, también presentó la mayor digestibilidad de 
la proteína con relación a los demás, mientras que el ENN fue menor para T1 $(p<0.05)$, las digestibilidades de grasa y fibra cruda fueron similares para todos los tratamientos (Tabla 5). En T1 se observó los más bajos $(p<0.05)$ NDT $(72.94 \%)$, ED (3.20 Mcal $/ \mathrm{kg} \mathrm{MS}$ ) y EM (2.59 Mcal $/ \mathrm{kg} \mathrm{MS})$ en comparación con el resto de tratamientos (Tabla 6).

Tabla 4. Consumo (C) y excreción (E) de nutrientes (gr) de los tratamientos

\begin{tabular}{|c|c|c|c|c|c|c|c|c|}
\hline Nutrientes & \multicolumn{2}{|c|}{ T1 } & \multicolumn{2}{|c|}{ T2 } & \multicolumn{2}{|c|}{ T3 } & \multicolumn{2}{|c|}{$\mathrm{T} 4$} \\
\hline & $\bar{C}$ & $\bar{E}$ & $\bar{C}$ & $\bar{E}$ & $\bar{C}$ & $\bar{E}$ & $\bar{C}$ & $\bar{E}$ \\
\hline $\begin{array}{l}\text { Materia } \\
\text { Seca }\end{array}$ & 86.11 & 20.5 & 84.56 & 15.35 & 75.84 & 13.45 & 74.43 & 10.34 \\
\hline Proteína & 16.49 & 1.79 & 15,29 & 1.77 & 12,59 & 1,52 & 11,15 & 0,67 \\
\hline Grasa & 8.87 & 0.20 & 7.96 & 0.58 & 7,27 & 0,55 & 7,19 & 0,36 \\
\hline Fibra cruda & 11.28 & 0.18 & 9.84 & 1.19 & 9,07 & 0,93 & 9,05 & 0,73 \\
\hline ENN & 48.21 & 3.30 & 42.85 & 8.54 & 39,29 & 8,38 & 38,95 & 6,47 \\
\hline
\end{tabular}

Tabla 5. digestibilidad asociada de los nutrientes (\%) en los tratamientos

\begin{tabular}{|c|c|c|c|c|} 
Nutrientes & $\mathrm{T} 1$ & $\mathrm{~T} 2$ & $\mathrm{~T} 3$ & $\mathrm{~T} 4$ \\
\hline Materia Seca & $76,19^{\mathrm{a}}$ & $81,85^{\mathrm{b}}$ & $82,27^{\mathrm{b}} \mathrm{c}$ & $86,11^{\mathrm{c}}$ \\
\hline Proteína & $50,81^{\mathrm{a}}$ & $88,37^{\mathrm{b}}$ & $87,93^{\mathrm{b}}$ & $93,99^{\mathrm{c}}$ \\
\hline Grasa & $90,02^{\mathrm{a}}$ & $92,67^{\mathrm{a}}$ & $92,42^{\mathrm{a}}$ & $94,95^{\mathrm{a}}$ \\
\hline Fibra cruda & $92,71^{\mathrm{a}}$ & $87,84^{\mathrm{a}}$ & $89,78^{\mathrm{a}}$ & $91,89^{\mathrm{a}}$ \\
\hline ENN & $68,92^{\mathrm{a}}$ & $80,05^{\mathrm{b}}$ & $78,66^{\mathrm{b}}$ & $83,39^{\mathrm{b}}$
\end{tabular}

Letras distintas en la misma fila son diferentes $(p<0.05)$

Las digestibilidades asociadas de la materia seca, la proteína y ENN fueron menores $(p<0.05)$ en $T 1$, mientras que en la grasa no se observó diferencias entre tratamientos, lo cual implica que los primeros componentes de las raciones afectan el aprovechamiento de estos nutrientes, demostrándose que el aumento de concentrado tiene una relación directamente proporcional con al incremento de los NDT y la digestibilidad asociada de la MS y la proteína, (Tablas 6 a 10 y Figura 1 a 4$)$.

En general las mezclas con diferentes proporciones de los dos alimentos fue positiva, siendo el porcentaje más bajo la digestibilidad de materia seca y la proteína en T1 contrario sucedió con el T4 donde con el incremento de 
concentrado se logró el índice de digestibilidad asociativa más elevado; convirtiéndose en una excelente opción a la hora de una implementación en producciones en donde se busque bajar los costos de alimentación disminuyendo el concentrado para reemplazarlo con el ramio que es de fácil obtención, buena calidad, y bajo costo para la zona de los llanos.

Tabla 6. Consumo (día/animal) y digestibilidad asociada de la Materia seca (MS) en los tratamientos

\begin{tabular}{|c|c|c|c|c|}
\hline Parámetros & T1 & T2 & T3 & $\mathrm{T} 4$ \\
\hline Consumo total de MS (gr) & $86.11^{\mathrm{b}}$ & $84.56^{b}$ & $75.84^{\mathrm{a}}$ & $74.43^{\mathrm{a}}$ \\
\hline Consumo de MS del ramio (gr) & $86,11^{d}$ & $68,56^{c}$ & $43,24^{b}$ & $24,43^{a}$ \\
\hline Consumo de concentrado (gr) & 0.00 & 16.00 & 32.00 & 48.00 \\
\hline $\begin{array}{l}\text { Proporción de concentrado en la ración } \\
\text { (gr) }\end{array}$ & 0.00 & $18.92^{\mathrm{a}}$ & $42.19^{b}$ & $64.49^{\mathrm{ac}}$ \\
\hline Proporción de ramio en la ración (\%) & $\underset{d}{100.00}$ & $81.08^{\mathrm{c}}$ & $57.81^{\mathrm{b}}$ & $35.51^{\mathrm{a}}$ \\
\hline $\begin{array}{l}\text { Digestibilidad asociada del ramio en la } \\
\text { ración (\%) }\end{array}$ & $76.19^{d}$ & $66.36^{c}$ & $47.56^{b}$ & $30.58^{a}$ \\
\hline $\begin{array}{l}\text { Digestibilidad asociada del concentrado } \\
\text { en la ración (\%) }\end{array}$ & 0.00 & $15.49^{a}$ & $34.71^{b}$ & $55.53^{c}$ \\
\hline NDT del ramio asociado a la ración (\%) & $72.94^{d}$ & $69.12^{c}$ & $48.37^{b}$ & $29.79^{a}$ \\
\hline $\begin{array}{l}\text { NDT del concentrado asociado a la } \\
\text { ración }(\%)\end{array}$ & 0.00 & 20.52 & $36.77^{b}$ & 59.19 \\
\hline NDT total de la ración (\%) & $72.94^{\mathrm{a}}$ & $89.64^{b}$ & $85.14^{b}$ & $88.98^{b}$ \\
\hline Energía digestible (Mcal $/ \mathrm{kg}$ & $3.20^{\mathrm{a}}$ & $3.94^{\mathrm{b}}$ & $3.74^{\mathrm{b}}$ & $3.91^{\mathrm{b}}$ \\
\hline Energía Metabolizable (Mcal/kg de MS & $2.59^{a}$ & $3.19^{b}$ & $3.03^{b}$ & $3.16^{b}$ \\
\hline
\end{tabular}

Letras distintas en la misma fila son diferentes $(p<0.05)$

En comparación con otros trabajos realizados (Takami, 1999), en donde se reporta una digestibilidad de $81 \%$ con ramio, se puede determinar que en la zona de piedemonte llanero el forraje posee una excelente calidad nutricional de fácil absorción y asimilación por el animal; en el estudio realizado en la sabana de Bogotá; se presumió que los suelos del piedemonte es donde este forraje tendría una mayor asimilación de nutrientes y por consiguiente una mayor expresión como suplemento al momento de administrarlo en una dieta para conejos en producción. 
Tabla 7. Consumo (día/animal) y digestibilidad asociada de la proteína en los tratamientos

\begin{tabular}{|l|c|c|c|c|}
\hline \multicolumn{1}{|c|}{ Parámetros } & T1 & T2 & T3 & T4 \\
\hline Consumo total de proteína (gr) & $16.49^{\mathrm{b}}$ & $15.29^{\mathrm{b}}$ & $12.59^{\mathrm{ab}}$ & $11.5^{\mathrm{a}}$ \\
\hline Consumo de proteína del ramio (gr) & $16.49^{\mathrm{c}}$ & $13,37^{\mathrm{c}}$ & $8,43^{\mathrm{b}}$ & $4,76^{\mathrm{a}}$ \\
\hline $\begin{array}{l}\text { Consumo de proteína del concentrado } \\
\text { (gr) }\end{array}$ & 0.00 & $1.92^{\mathrm{a}}$ & $4.16^{\mathrm{b}}$ & $6.39^{\mathrm{c}}$ \\
\hline Proporción de ramio en la ración (\%) & $100^{\mathrm{d}}$ & $87.44^{\mathrm{c}}$ & $66.97^{\mathrm{b}}$ & $42.73^{\mathrm{a}}$ \\
\hline $\begin{array}{l}\text { Proporción de concentrado en la ración } \\
\text { (\%) }\end{array}$ & 0,00 & $12.56^{\mathrm{a}}$ & $33.03^{\mathrm{b}}$ & $57.27^{\mathrm{c}}$ \\
\hline $\begin{array}{l}\text { Digestibilidad asociada del ramio en la } \\
\text { ración (\%) }\end{array}$ & 50.81 & $77.27^{\circ}$ & 58,89 & $40,16^{\mathrm{a}}$ \\
\hline $\begin{array}{l}\text { Digestibilidad asociada del concentrado } \\
\text { en la ración (\%) }\end{array}$ & 0.00 & $11.10^{\mathrm{a}}$ & $29.04^{\mathrm{b}}$ & $53.83^{\mathrm{c}}$ \\
\hline $\begin{array}{l}\text { Digestibilidad asociada total en la ración } \\
\text { (\%) }\end{array}$ & $50.81^{\mathrm{a}}$ & $88.37^{\mathrm{b}}$ & $87.93^{\mathrm{b}}$ & $93.43^{\mathrm{b}}$ \\
\hline
\end{tabular}

Letras distintas en la misma fila son diferentes $(p<0.05)$.

Tabla 8. Consumo (día/animal) y digestibilidad asociada de la grasa en los tratamientos

\begin{tabular}{|l|c|c|c|c|}
\hline \multicolumn{1}{|c|}{ Parámetros } & $\mathrm{T} 1$ & $\mathrm{~T} 2$ & $\mathrm{~T} 3$ & $\mathrm{~T} 4$ \\
\hline Consumo total de grasa (gr) & $8,87^{\mathrm{b}}$ & $7,96^{\mathrm{ab}}$ & $7,27^{\mathrm{a}}$ & $7,19^{\mathrm{a}}$ \\
\hline Consumo de grasa del ramio (gr) & $8,87^{\mathrm{d}}$ & $6,31^{\mathrm{c}}$ & $3,98^{\mathrm{b}}$ & $2,25^{\mathrm{a}}$ \\
\hline Consumo de grasa del concentrado (gr) & 0 & $1,65^{\mathrm{a}}$ & $3,29^{\mathrm{b}}$ & $4,94^{\mathrm{c}}$ \\
\hline $\begin{array}{l}\text { Proporción de concentrado en la ración } \\
\text { (\%) }\end{array}$ & 100 & $79,24^{\mathrm{a}}$ & $54,72^{\mathrm{b}}$ & $31,26^{\mathrm{a}}$ \\
\hline $\begin{array}{l}\text { Proporción de concentrado en la ración } \\
\text { (\%) }\end{array}$ & 0 & $20,76^{\mathrm{a}}$ & $45,28^{\mathrm{b}}$ & $68,74^{\mathrm{c}}$ \\
\hline $\begin{array}{l}\text { Digestibilidad asociada del ramio en la } \\
\text { ración (\%) }\end{array}$ & $90,02^{\mathrm{d}}$ & $73,43^{\mathrm{c}}$ & $50,57^{\mathrm{b}}$ & $29,68^{\mathrm{a}}$ \\
\hline $\begin{array}{l}\text { Digestibilidad asociada del concentrado } \\
\text { en la ración (\%) }\end{array}$ & 0,00 & $19,24^{\mathrm{a}}$ & $41,85^{\mathrm{b}}$ & $65,27^{\mathrm{bc}}$ \\
\hline $\begin{array}{l}\text { Digestibilidad asociada total en la ración } \\
\text { (\%) }\end{array}$ & $90.02^{\mathrm{a}}$ & $92.67^{\mathrm{a}}$ & $92.42^{\mathrm{a}}$ & $94.95^{\mathrm{a}}$ \\
\hline
\end{tabular}

Letras distintas en la misma fila son diferentes $(p<0.05)$.

Al momento de la comparación entre el ramio y el concentrado dentro de una producción se determinó que con el uso de este forraje se necesita una mayor cantidad de alimento por ración, ya que el concentrado posee un mayor porcentaje de nutrientes que el ramio en: grasa, fibra cruda y ENN. Sin embargo, el \% NDT $(72,94 \%)$ del ramio indica su buena calidad nutricional, con la ventaja de ser una 
fuente alimenticia económica, de fácil obtención, buena palatabilidad, Factores que fueron evaluados positivamente en la elaboración de dietas comerciales para la alimentación de conejos (De Blas et al., 1985 y Nieves et al. 2004).

Tabla 9. Consumo (día/animal) y digestibilidad asociada de la fibra cruda (FC) en los tratamientos

\begin{tabular}{|l|c|c|c|c|}
\hline \multicolumn{1}{|c|}{ Parámetros } & $\mathrm{T} 1$ & $\mathrm{~T} 2$ & $\mathrm{~T} 3$ & $\mathrm{~T} 4$ \\
\hline Consumo total de fibra cruda (gr) & $11,28^{\mathrm{b}}$ & $9,84^{\mathrm{a}}$ & $9,07^{\mathrm{a}}$ & $9,05^{\mathrm{a}}$ \\
\hline $\begin{array}{l}\text { Consumo de fibra cruda del ramio } \\
\text { (gr) }\end{array}$ & $11,28^{\mathrm{d}}$ & $7,75^{\mathrm{c}}$ & $4,89^{\mathrm{b}}$ & $2,76^{\mathrm{a}}$ \\
\hline $\begin{array}{l}\text { Consumo de fibra cruda del } \\
\text { concentrado (gr) }\end{array}$ & 0 & $2,09^{\mathrm{a}}$ & $4,18^{\mathrm{b}}$ & $6,29^{\mathrm{c}}$ \\
\hline Proporción de ramio en la ración (\%) & $100^{\mathrm{d}}$ & $78,73^{\mathrm{c}}$ & $53,87^{\mathrm{b}}$ & $30,50^{\mathrm{a}}$ \\
\hline $\begin{array}{l}\text { Proporción de concentrado en la } \\
\text { ración (\%) }\end{array}$ & 0 & $21,27^{\mathrm{a}}$ & $46,13^{\mathrm{b}}$ & $69,50^{\mathrm{c}}$ \\
\hline $\begin{array}{l}\text { Digestibilidad asociada del ramio en } \\
\text { la ración (\%) }\end{array}$ & $92,91^{\mathrm{d}}$ & $69,16^{\mathrm{c}}$ & $48,37^{\mathrm{b}}$ & $28,03^{\mathrm{a}}$ \\
\hline $\begin{array}{l}\text { Digestibilidad asociada del } \\
\text { concentrado en la ración (\%) }\end{array}$ & 0,00 & $18,68^{\mathrm{a}}$ & $41,41^{\mathrm{b}}$ & $63,86^{\mathrm{c}}$ \\
\hline $\begin{array}{l}\text { Digestibilidad asociada total en la } \\
\text { ración (\%) }\end{array}$ & $92,91^{\mathrm{a}}$ & $87.84^{\mathrm{a}}$ & $89.78^{\mathrm{a}}$ & $91.89^{\mathrm{a}}$ \\
\hline
\end{tabular}

Letras distintas en la misma fila son diferentes $(p<0.05)$.

Tabla 10. Consumo (día/animal) y digestibilidad asociada de extracto no nitrogenado (ENN) en los tratamientos

\begin{tabular}{|c|c|c|c|c|}
\hline Parámetros & T1 & T2 & T3 & T4 \\
\hline Consumo total de ENN (gr) & $48,2^{c}$ & $42,85^{a b}$ & $39,29^{a b}$ & $38,95^{a}$ \\
\hline Consumo de ENN del ramio (gr) & $42,57^{d}$ & $33,90^{c}$ & $21,38^{b}$ & $12,08^{a}$ \\
\hline $\begin{array}{l}\text { Consumo de ENN del concentrado } \\
\text { (ar) }\end{array}$ & 0 & $8,95^{\mathrm{a}}$ & $17,91^{b}$ & $87^{d}$ \\
\hline $\begin{array}{l}\text { Proporción de concentrado en la } \\
\text { ración (\%) }\end{array}$ & $100^{d}$ & $79,10^{c}$ & 54 , & $31,01^{a}$ \\
\hline de concentrado en la & 0 & $20,90^{a}$ & 45 , & $68,99^{\circ}$ \\
\hline $\begin{array}{l}\text { ilidad asociada del ramio en } \\
n(\%)\end{array}$ & $68,92 \mathrm{~d}$ & $63,32^{c}$ & & $25,86^{a}$ \\
\hline $\begin{array}{l}\text { Digestibilidad asociada del } \\
\text { concentrado en la ración (\%) }\end{array}$ & 0,00 & $24,52^{\mathrm{a}}$ & 35 & $57,53^{c}$ \\
\hline $\begin{array}{l}\text { Digestibilidad asociada total en la } \\
\text { ración (\%) }\end{array}$ & $68,92^{a}$ & $87.84^{c}$ & $78.66^{b}$ & $83.39^{c}$ \\
\hline
\end{tabular}

Letras distintas en la misma fila son diferentes $(p<0.05)$. 


\section{CONCLUSIÓN}

La palatabilidad del ramio es excelente lo que conlleva a que tenga un mayor consumo por parte del animal; convirtiéndolo en una opción como fuente básica de alimento en cunicultura. Es importante tener presente que al momento de suministrar ramio en la dieta para conejos es aconsejable hacerla con una proporción de concentrado para lograr un efecto asociativo positivo de ingredientes, puesto que el contenido nutricional del concentrado eleva el aprovechamiento de los nutrientes del ramio, especialmente el de MS y proteína.
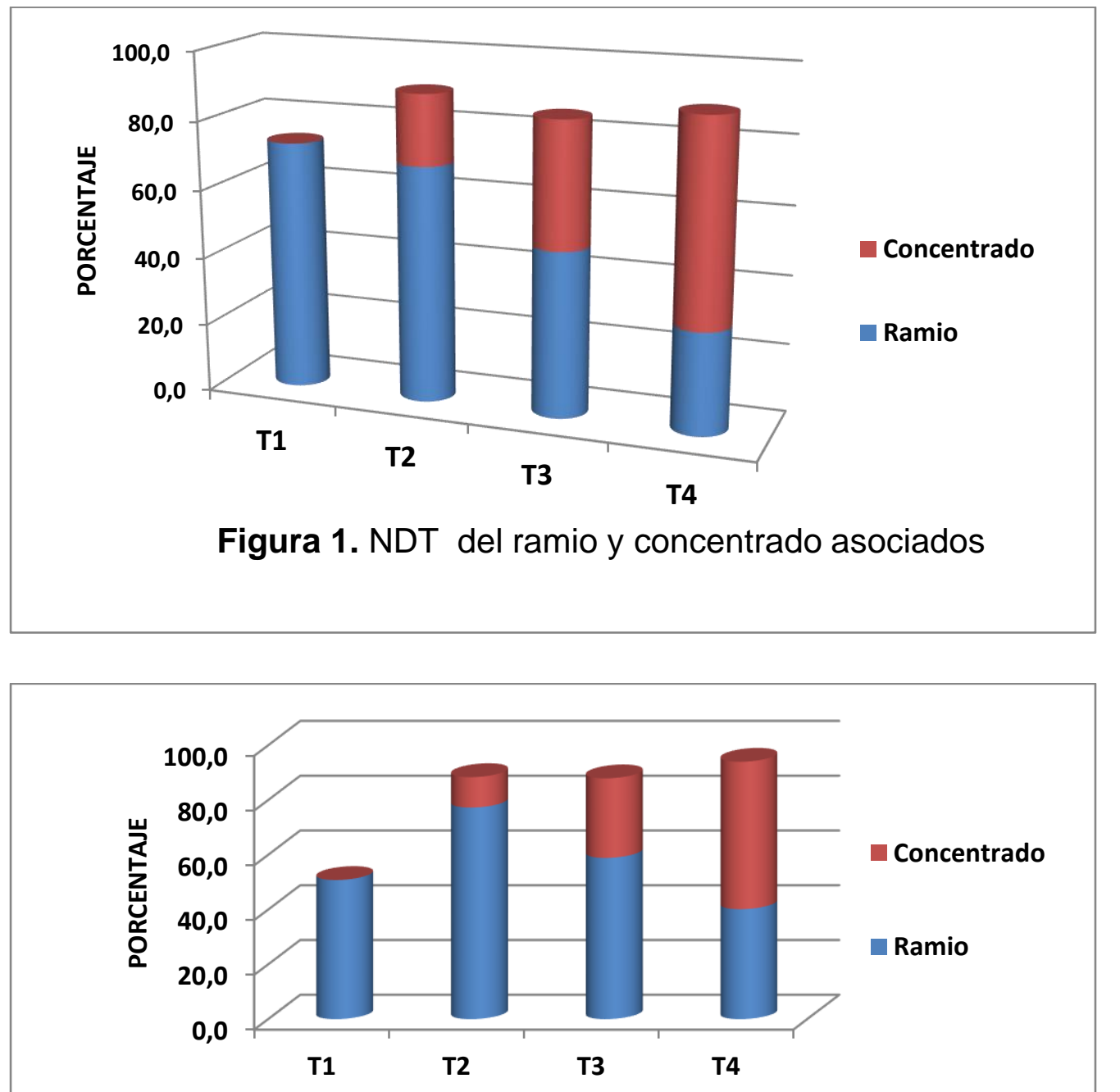

Figura 2 . Digestibilidad de la proteína asociada 


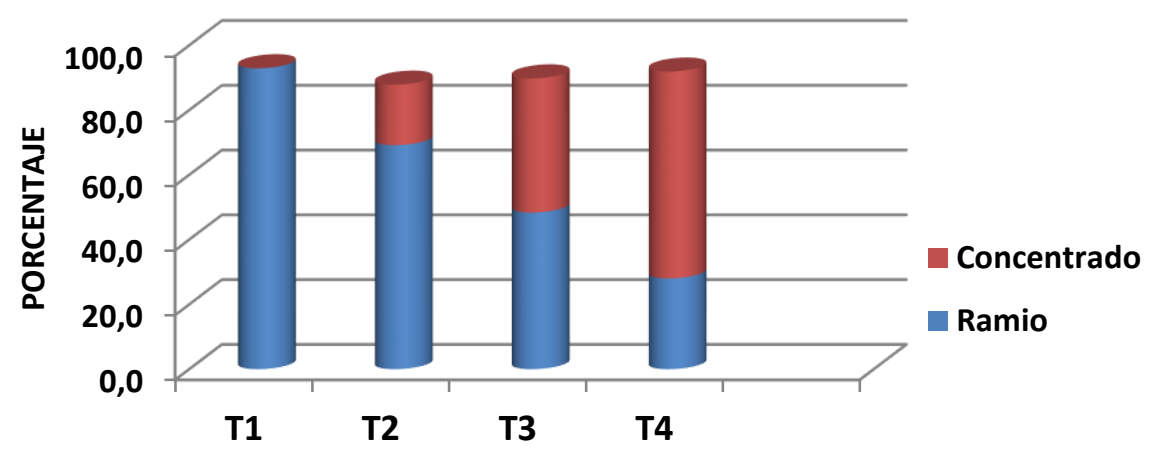

Figura 3. Digestibilidad asociada de la fibra cruda

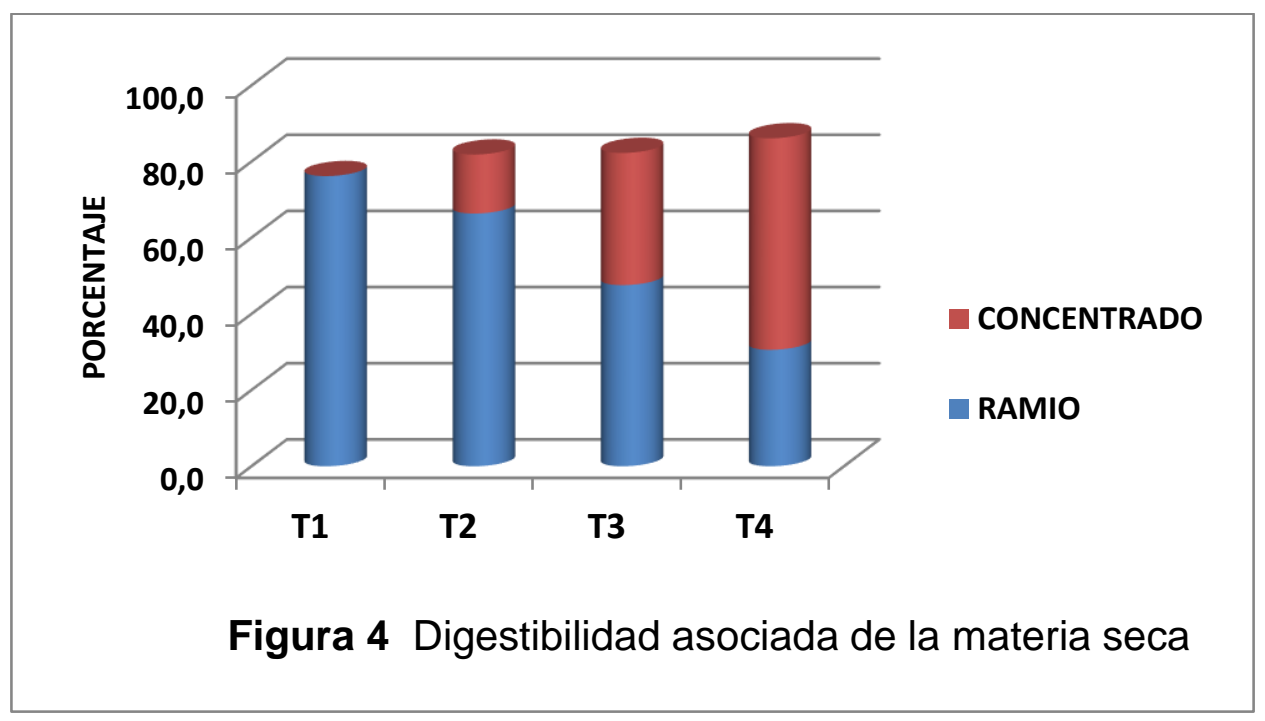

\section{BIBLIOGRAFÍA}

1. AOAC. Official Methods of Analysis (18 $\left.{ }^{\text {th }}\right)$ Association of Official Analytical Chemists, Arligton, VA. Washington, D.C. 2006.

2. Castellanos R. A., Llamas L. G., Shimada A. 1990. Manual de técnicas de investigación en ruminología. México. 1990: 29-34.

3. Cheeke P R. Rabbit Feeding and Nutrition. Orlando: Academic Press, 1987.

4. De Blas C, Fraga M, Rodríguez J. Units for feed evaluation and requeriments for commercially grown rabbits. J Anim. Sci. 1985: 60: 1021-1028.

5. Church D.C.; Pond W.G. Fundamentos de nutrición y alimentación de animales. $2^{\text {a }}$ ed. Ed. Limusa. México. 1990: 438

6. Jenkins J. Feeding Recommendations for the house rabbit. The Veterinary Clinics of North America, 1999.

7. Mundo Pecuario. Método de los nutrientes digestibles totales NDT. [Consultado 10-10-2011] Disponible: http://mundo- 
pecuario.com/tema73/valoracion energetica raciones animales/nutrientes di gestibles totales-859.html

8. National Research Council. Nutrient requirements of rabbits, Second Revised Edition, Committee on Animal nutrition. 1977: 30.

9. Nieves D, López, D, Cadena. Alimentación de conejos de engorde con dietas basadas en materias primas no convencionales y suplementación con Trichanthera gigantea Rev Cien Tec. 2001: 60-66.

10. Nieves D, Terán O, Silva L, González C. Digestibilidad in vivo de nutrientes en dietas en forma de harina con niveles crecientes de Leucaena leucocephala para conejos de engorde. Rev Cient. 2002: 408-411.

11. Nieves D, Cordero J, Teran O. Aceptabilidad de dietas con niveles crecientes de morera (Morus alba) en conejos destetados. Zootecnia Trop., 2004: 22 (2): 202-209.

12. Nieves, Do, Araque H, Teran. Digestibilidad de nutrientes del follaje de morera (Morus alba) en conejos de engorde. Rev. Cient. 2006: 16 (4): 315-324.

13. Nieves D, Barajas A, Delgado G. Digestibilidad fecal de nutrientes en dietas con forrajes tropicales en conejos: Comparación entre métodos directo e indirecto. Bioagro, 2008: 20 (1): 73-75.

14. Olivo SR. Evaluación del comportamiento productivo y reproductivo del conejo (Cavia porcellus) criollo mejorado Conocoto-Pichincha. Universidad Central de Quito, Facultad de Ciencias Agrícolas. Tesis Ing. Agr. Pág. 78. 1989.

15. Preston T, Rodríguez I, Van L, Ha chau I. Follaje de la yuca (Manihot esculenta Cranz) como fuente de proteína para la producción animal en sistemas agroforestales. Conferencia de la FAO sobre "agroforesteria para la producción animal en Latinoamérica". 1999.

16. Roa M L, Muñoz J, Céspedes DA. Evaluación nutricional de tres especies de árboles forrajeros en bovinos fistulados. Colombia Revista Acovez 1999: 24: 14-18.

17. Tamaki HR. Prueba de dos niveles de vitamina $C$ como posible sustituto del forraje verde en la alimentación de conejos. Tesis. Universidad Nacional Agraria. Pág. 86. 1999. 